\title{
Cuidado do idoso dependente no contexto familiar: uma revisão de literatura
}

\author{
Marina Jede*, Mariana Spuldaro*
}

\section{Resumo}

A família é considerada o habitat natural da pessoa. É nela que somos conhecidos pelos nossos defeitos, qualidades e necessidades. Nesse sentido, podemos considerar importante o relacionamento do idoso com a família em qualquer fase da vida, em especial quando eles se tornam dependentes física e psicologicamente. Diante disso, objetivou-se verificar de que forma está ocorrendo o cuidado do idoso dependente no contexto da família. Tratase de um estudo de revisão bibliográfica utilizando os artigos nacionais da base de dados Scielo, empregando-se os descritores cuidado, idoso dependente, família, envelhecimento, saúde do idoso. Também foram usados teses, dissertações e capítulos de livros de gerontologia publicados no período de 1987 a 2007. Emergiram as seguintes categorias temáticas: necessidades do idoso dependente do cuidado familiar, disponibilidade da família para realizar o cuidado do idoso dependente. O grau de dependência influencia diretamente na vida do idoso e de sua família, pois a dificuldade de realizar as atividades da vida diária gera impacto na estrutura familiar. A assistência no domicílio contribui para humanização do cuidado de forma mais efe- tiva e participativa. $\mathrm{O}$ apoio ao cuidador e a educação em saúde são fundamentais para orientá-lo e auxiliá-lo nas situações mais difíceis do cuidado.

Palavras-chave: Cuidado. Idoso dependente. Família. Envelhecimento. Saúde do idoso.

\section{Introdução}

Na população idosa, muitas vezes os valores culturais são marcados pelo importante papel da família. A família é considerada o habitat natural da pessoa. Assim, podemos considerar que o relacionamento do idoso com a família é importante em qualquer fase da vida.

Os idosos são bastante vulneráveis; por isso apresentam uma combinação de limitações e doenças que reduzem sua capacidade funcional e, em consequência disso, acabam atingindo o seu emocional.

O medo de envelhecer e a incapacidade de muitos de se confrontar com seu próprio processo de envelhecimento

* Acadêmicas do curso de Enfermagem da Universidade de Passo Fundo. Trabalho desenvolvido na disciplina de Iniciação Científica do curso de Enfermagem da Universidade de Passo Fundo. Endereço para correspondência: Av. Barão do Rio Branco, n. 187. CEP 99150-000, Marau - RS. E-mail: marianaspuldaro@yahoo. com.br

$\hookrightarrow$ Recebido em março de 2009 - Avaliado em agosto de 2009.

$\rightarrow$ doi:10.5335/rbceh.2009.040 
podem levar ao preconceito e à discriminação contra a pessoa idosa. Para Smeltzer e Bare (2005), só pela compreensão desse processo e pelo respeito à pessoa como um indivíduo é que os mitos do envelhecimento podem ser eliminados. Se os idosos forem tratados com dignidade e encorajados a manter a autonomia, sua qualidade de vida irá melhorar.

A velhice acarreta a diminuição da capacidade de adaptação, que ocorre de maneira objetiva, limitando o sistema funcional e, de uma maneira mais evidente, o sistema psicossocial, no qual se manifesta pela dificuldade de aceitação. Isso tudo leva ao aumento da dependência do ambiente familiar, que é um local de proteção e estabilidade.

O papel da família é fundamental no cuidado do idoso. A família predomina como alternativa no sistema de suporte informal aos idosos, afirma Caldas (2003), mas é preciso destacar que, embora o cuidado familiar seja um aspecto importante, não atinge todos os idosos.

$\mathrm{Na}$ verdade, a população idosa é proveniente de uma época com marcados valores culturais, na qual a família exercia importante papel. Para os familiares cuidar dos doentes era honroso, considerando a família centro de intimidade e centro de abertura em relação ao cuidado do idoso. (LEME; SILVA, 2002).

O contato com o idoso doente, segundo Leme e Silva (2002), só será completo com a presença da família, em razão da sua estrutura, potencialidades e capacidades. Como o conceito de saúde para OMS é o estado de bem-estar físico, psíquico e social, será fácil perceber que para caracterizar o idoso sadio e idoso doente é importante a caracterização de sua situação familiar.

O cuidado de forma inadequada, ineficiente, ou mesmo inexistente, é observado em situações nas quais os membros da família não estão disponíveis, estão sobrecarregados ou despreparados para essas responsabilidades. Nesses casos, existe a possibilidade de maus-tratos e abuso. Portanto, por mais que a legislação, as políticas públicas e até mesmo a sociedade afirmem e acreditem que os idosos devem ser cuidados pela família, não pode garantir que esta prestará um cuidado humanizado. Para acompanhar essas situações são necessários programas e serviços para idosos. Essas ações são urgentes e importantes, pois muitos idosos isolados, dependentes e abandonados necessitam de alternativas à assistência familiar de que não dispõem. (CALDAS, 2003).

Em decorrência disso, a família cuidadora necessita de informação sobre como realizar os cuidados e adaptação do idoso. É muito importante o suporte emocional, que garante qualidade de vida aos cuidadores.

Segundo Karsh (2003), é preciso chamar a atenção dos profissionais de saúde que prestam cuidados em casa a idosos dependentes, para que sejam consideradas as mudanças sociais e econômicas que estão transformando as estruturas familiares nas cidades brasileiras e como essas podem afetar a posição e o papel tradicional do cuidador de idosos fragilizados e dependentes.

Os cuidados implementados pela família têm o objetivo de preservar a vida de seus membros para alcançar o 
desenvolvimento pleno de suas potencialidades, de acordo com suas próprias possibilidades e com as condições do meio em que ela vive. (ELSEN; MARCON; SANTOS, 2002).

Para Caldas (2003), a família apresenta necessidades que vão desde os aspectos materiais, emocionais e também necessidade de informações. Além disso, é importante o suporte emocional, uma rede de cuidados que une a família aos serviços de apoio e meios que garantam qualidade de vida aos cuidadores.

Conforme Leal (2000), o cuidador familiar é aquele que põe a necessidade do outro em primeiro lugar. Geralmente, é tão pressionado por necessidades imediatas que se esquece de si mesmo. Em muitos casos, o cuidador é também uma pessoa frágil, já em idade de envelhecimento ou com possibilidade de adoecimento. Logo, o cuidador sem suporte pode ser o futuro paciente.

Acredita-se que este estudo é de suma importância, pois estará contribuindo para atualizar o conhecimento sobre o processo de envelhecer com dependência familiar, já que esse assunto, em razão do crescimento acelerado da população idosa, está cada vez mais presente na realidade da população.

Diante disso, objetivou-se verificar de que forma está ocorrendo o cuidado do idoso dependente no contexto da família, descrito na literatura.

\section{Metodologia}

Trata-se de um estudo de revisão bibliográfica utilizando-se a Biblioteca Virtual em Saúde (BVS) e os descritores cuidado e idoso dependente. Foram acessadas 873 fontes, as quais foram refinadas por outro descritor (família) e por idioma (português), resultando em quatro fontes. Limitou-se o período de 2002 a 2006.

Também foram utilizados artigos publicados no idioma português e capítulos de livros de gerontologia no período de 1995 a 2007, localizados em biblioteca convencional. Dessa forma, foram selecionadas para o estudo oito fontes.

Primeiramente, foi realizada uma leitura exploratória para verificar se a obra consultada interessava à pesquisa. Após essa leitura, procedia-se à leitura seletiva do material que de fato interessava. Por fim, era feita a leitura analítica, com a finalidade de analisar as fontes, de forma a possibilitar a obtenção de respostas ao problema da pesquisa.

A análise das fontes bibliográficas selecionadas possibilitou a elaboração das seguintes categorias temáticas: necessidades do idoso dependente do cuidado familiar e disponibilidade da família para realizar o cuidado do idoso dependente.

Necessidades do idoso dependente do cuidado familiar

No Brasil, a velhice sem independência e autonomia faz parte de uma face oculta da opinião pública, porque vem sendo mantida no ambiente familiar dos domicílios ou nas instituições, impedindo qualquer nitidez e, consequentemente, qualquer preocupação política de proteção social.

O idoso fragilizado, bem como o seu cuidador, que inúmeras vezes também é idoso, é apegado ao seu ambiente e 
objetos pessoais. A mudança da moradia, do lar, o distanciamento dos conhecidos podem gerar depressão e desencadear outras doenças. Esses dados remetem à preocupação por mais de seis milhões de pessoas e famílias e a um e meio milhão de idosos gravemente fragilizados no Brasil, segundo a Pesquisa Nacional por Amostra de Domicílios (PNAD) de 2001. (IBGE, 2002).

Dessa forma, o envelhecimento com dependência gera significados particulares, que, dentro de um cenário histórico, social, político, econômico e cultural, precisam ser analisados e esclarecidos. (KARSCH, 2003).

Em relação à autonomia da pessoa idosa, está ligada diretamente a sua capacidade funcional, principalmente no que diz respeito às atividades da vida diária, o que proporciona ao idoso a sensação de bem-estar ou uma boa qualidade de vida, independentemente de sua idade. (RODRIGUES; WATANABE; DERNTL, 2006).

De acordo com os conceitos gerontológicos, o idoso que mantém a sua autodeterminação, sem necessitar de nenhum tipo de ajuda ou supervisão para realizar seus afazeres diários, é considerado um idoso saudável, ainda que possua uma ou mais doenças crônicas. Daí decorre o conceito de "capacidade funcional", ou seja, a capacidade de manter as habilidades físicas e mentais necessárias para uma vida independente e autônoma. A determinação da capacidade funcional do idoso é um indicador imprescindível para adequar os cuidados de enfermagem tanto ao paciente como ao familiar. A família é fundamental nesse processo de prestação de cuidados ao idoso e deve ser compreendida quando os cuidados excedem as suas capacidades. (THOBER; CREUTZBERG; VIEGAS, 2005).

Estudos revelam que cerca de $40 \%$ dos indivíduos com 65 anos ou mais de idade precisam de algum tipo de ajuda para realizar, pelo menos, uma tarefa, como fazer compras, cuidar das finanças, preparar refeições e limpar a casa. Uma parcela menor (10\%) requer auxílio para realizar tarefas básicas, como tomar banho, vestir-se, ir ao banheiro, alimentarse, sentar-se e levantar-se de cadeiras e camas. (KARSCH, 2003). A cronicidade dessa situação e o próprio avanço da idade acabam levando a um estado de incapacidade e dependência para os cuidados nas atividades da vida diária.

$\mathrm{O}$ envelhecimento dependente e o cuidador exigem novas formas de assistência por parte das políticas públicas de saúde. A população de baixa renda procura apoio a essa realidade de exclusão social do idoso quando esse perde sua capacidade funcional. (KARSCH, 2003).

Há necessidade de participação consciente e ativa dos familiares, porque muitos negam o declínio funcional e psicológico do idoso quando este apresenta mais de uma doença sem bom prognóstico, que eles desconhecem na maioria das vezes, gerando uma postura de indiferença e dificuldade na relação idoso-família.

Quando o idoso se torna dependente, as alterações são inevitáveis e envolvem afeto, finanças, relações de poder e outras, desenvolvendo um processo de reorganização familiar.

A dependência leva um tempo para ser compreendida pelo familiar, dificul- 
tando a tomada de decisões em relação ao cuidado. (THOBER; CREUTZBERG; VIEGAS, 2005).

A perda de independência, e até da autonomia de um idoso, pressupõe que, em casa, alguém assuma as funções de cuidador e isso gera a dificuldade de mobilidade.

As dificuldades vêm desde a falta de condicionamento físico para realizar a movimentação até o medo de não conseguir realizar esse movimento de forma adequada. A habilidade e o conhecimento da atividade de cuidar são construídos na prática diária, na qual o familiar aprende com os seus erros e acertos, seguindo um caminho inverso da trajetória profissional, o qual primeiro tem contato com o conhecimento e é treinado para só depois, estar habilitado para exercer a atividade. (THOBER; CREUTZBERG; VIEGAS, 2005).

Disponibilidade da família para realizar 0 cuidado do idoso dependente

O cuidar é a verdadeira atenção à saúde da pessoa humana, enquanto conceituada como estado de bem-estar físico, psíquico e social. Compreende não apenas a busca da cura das doenças, mas o apoio e a paliação quando a cura já não é possível e, finalmente, o apoio para o fim da vida sem dores e sem sofrimento desnecessário, preservando a dignidade da pessoa humana. (BRUM; TOCANTINS; SILVA, 2005).

Diferentes países do mundo têm desenvolvido formas de apoio e cuidados aos seus idosos dependentes e, em alguns países, o suporte oferecido é quase exclusivamente de responsabilidade do
Estado; em outros, são predominantemente as famílias que desempenham todos os cuidados. (KARSCH, 2003; CREUTZBERG, 2000).

Estudos realizados no Brasil e no mundo constatam que os idosos dependentes recebem o apoio de uma ou mais pessoas dentre familiares, especialmente de mulheres que residem no mesmo domicílio e se tornam as cuidadoras de seus maridos, pais e irmãos. (THOBER; CREUTZBERG; VIEGAS, 2005).

As maiores mudanças do cotidiano acontecem na vida pessoal e profissional do próprio cuidador. O cuidado constante exige do cuidador praticamente todo o seu tempo, tanto que alguns cuidadores abdicam do seu emprego, outros trabalham, mas carregam a preocupação com a saúde do seu familiar. (THOBER; CREUTZBERG; VIEGAS, 2005).

Enquanto para alguns cuidadores a rotina de cuidar é algo que pode se tornar exaustivo e comprometer sua integridade física, outros continuam a exercer sua atividade profissional não transformando o cuidado na sua ocupação.

Uma das principais dificuldades encontradas é a escassez de recursos financeiros, com os quais é possível obter recursos humanos e materiais. A falta de dinheiro pode gerar angústia do cuidador que quer dar o melhor para o seu familiar idoso. (THOBER; CREUTZBERG; VIEGAS, 2005).

O aumento do grau de dependência do idoso gera para o cuidador a dificuldade na mobilidade. As dificuldades vão desde a falta de condicionamento físico para realizar a movimentação até o medo de não conseguir realizar o movimento 
de forma adequada. A habilidade e o conhecimento da atividade de cuidar são construídos na prática diária, na qual o familiar aprende com os seus erros e acertos, seguindo um caminho inverso da trajetória profissional. A falta de preparo para o cuidado gera no cuidador uma ansiedade que é substituída por segurança a partir do momento em que consegue se organizar e perceber $o$ cuidado como fácil.

O processo de cuidar do idoso em contexto domiciliar pode desencadear o aparecimento de limitações na vida cotidiana do cuidador, com consequente risco à sua saúde e bem-estar, como, por exemplo, cansaço, depressão, conflitos com o cônjuge, aumento dos problemas econômicos, entre outros. (GONÇALVES et al., 2006).

$\mathrm{O}$ ambiente doméstico passa a ser adaptado para as necessidades emergentes. Objetos de decoração passam a ser substituídos por medicações, materiais de curativo, uma série de objetos que fazem parte do cuidado. As adaptações facilitam o cuidado, mas tornam o ambiente menos aconchegante.

O cuidado também é percebido como gratidão ao familiar idoso e zelo por seu bem-estar. O cuidar, quando se torna gratificante, é uma demonstração de reconhecimento pela família e algumas vezes pelo idoso. (THOBER; CREUTZBERG; VIEGAS, 2005).

A assistência domiciliar contribui para a humanização da assistência, buscando envolver o familiar no cuidado e na construção de um ambiente favorável à recuperação. $\mathrm{O}$ processo de educação em saúde acontece de forma mais efetiva e participativa se desenvolvido no domicílio. A assistência domiciliária ao idoso não beneficia somente a este, mas contribui para melhorar a qualidade de vida também dos familiares. Essa assistência demanda dos profissionais da saúde uma parceria com as pessoas que cuidam dos idosos, possibilitando a sistematização das tarefas a serem realizadas no próprio domicílio, dando maior atenção àquelas relacionadas à promoção da saúde, à prevenção de incapacidades e à manutenção da capacidade funcional do idoso dependente e do seu cuidador, evitandose, assim, na medida do possível, hospitalizações, asilamentos e outras formas de segregação e isolamento. (BRASIL, 2002; CREUTZBERG, 2000).

Também merece destaque que o cuidar envolve uma atitude do profissional integrado por duas formações: a pessoal e profissional. As possíveis repercussões desses valores, com reflexo na prática dos enfermeiros, podem ser percebidas no cotidiano no relacionamento entre pacientes-profissionais de enfermagem. Esse relacionamento perpassa pela subjetividade do profissional que assiste, intervindo no cuidar. (BRUM; TOCANTINS; SILVA, 2005).

Para o cuidador familiar a rotina de cuidar é algo que pode se tornar exaustivo e comprometer sua integridade física. Diversos motivos contribuem para que uma pessoa se torne cuidadora principal, dentre os quais se destacam: a obrigação moral alicerçada em aspectos culturais e religiosos; a condição de conjugalidade, o fato de ser esposo ou esposa; a ausência de outras pessoas para a tarefa do cuidar, caso em que o cuidador assume 
essa incumbência não por opção, mas, geralmente, por força das circunstâncias; as dificuldades financeiras, como em caso de filhas desempregadas que cuidam dos pais em troca do sustento. (GONÇALVES et al., 2006).

Para os cuidadores as palavras "cuidar" e "cuidado" fundem-se no mesmo significado, tanto em termos de definição como pela identificação das atividades que o envolvem. As qualidades e habilidades do cuidador, geralmente, são adquiridas pelas necessidades emergentes do dia a dia. Muitos cuidadores aprenderam com as dificuldades a adaptar a sua vida e o ambiente para o cuidado. (THOBER; CREUTZBERG; VIEGAS, 2005).

A atitude do idoso em relação ao cuidador pode muitas vezes interferir na forma como este é tratado. O cuidador sofre influência da personalidade e caráter do idoso e do relacionamento durante o decorrer dos anos. Percebeu-se que, se o idoso trata com desprezo, é tratado dessa forma: se trata com carinho, dessa forma também será tratado. A forma negativa como o idoso trata o cuidador, de certa forma, pode ser compreendida como não aceitação da relação de dependência. Isso ocorre principalmente na relação entre cônjuges, quando o marido passa a depender da sua esposa, a qual precisa assumir a total responsabilidade. (THOBER; CREUTZBERG; VIEGAS, 2005).

O cuidador familiar de idosos incapacitados precisa ser alvo de orientação sobre como proceder nas situações mais difíceis e receber em casa periódicas visitas de profissionais. Este apoio é fundamental quando se trata de um casal de idosos, em que o cônjuge menos lesado assume os cuidados do outro que foi acometido por uma súbita e grave doença incapacitante. (KARSCH, 2003).

A família é fonte de apoio. Cada parte colabora e dá a sua contribuição para o cuidado, seja no cuidado direto ao familiar ou apoiando, seja se importando com o que tem sido feito. Esse tipo de apoio é muito significativo.

Tudo o que existe e vive precisa ser cuidado para continuar a existir e a viver. A essência do ser humano reside no cuidado. $O$ cuidado é mais fundamental do que a razão e a vontade. (SOUZA; VAZ, 2007).

\section{Considerações finais}

O envelhecimento da população é um fenômeno relativamente recente e irreversível, e a dependência desse grupo vem sendo abordada cada vez mais. $\mathrm{O}$ grau de dependência influencia diretamente na vida do idoso e de sua família, pois a dificuldade de realizar as atividades da vida diária determinam a necessidade de um cuidador que se disponibilize a auxiliá-lo. O conhecimento da realidade das famílias do ponto de vista de suas necessidades, das dificuldades e facilidades geradas no cotidiano, como também os sentimentos que envolvem o cuidado, é fundamental para a assistência dos profissionais. A dependência de um familiar idoso gera impacto na dinâmica, na economia e na saúde da família que se ocupa dos cuidados.

A assistência domiciliar contribui para a humanização, buscando envolver o familiar na construção de um ambiente 
favorável à recuperação. $\mathrm{O}$ processo de educação em saúde acontece de forma mais efetiva e participativa se desenvolvido no domicilio. É fundamental o apoio ao cuidador familiar de idosos incapacitados, o qual precisa ser alvo de orientações sobre como proceder nas situações mais difíceis do cuidado. $\mathrm{O}$ envelhecimento com dependência toma significados particulares num contexto social, econômico e cultural. Assim, cada parte colabora e contribui para 0 cuidado.

The dependent aging care in the familiar context: a literature review

\section{Abstract}

The family is considered the natural habitat of the person. In it are known by our defects, skill and needs. In this sense, we can considerate important relationship between aging and his family in any phase of the life, especially when they become physically and psychologically dependent. In this context, it is very important to che$\mathrm{ck}$ that is happening with the care of aging in the family. It was a bibliographical review study, using the national text as based data Scielo, being used the items taken care, dependent senior, family, aging, the senior's health. Also theories, dissertations and chapters of gerontology books were used published in the period from 1987 to 2007. Emerged these thematic categories: familiar care of dependent senior needs, availableness of family take care the dependent senior. The degrees of dependent influence directly the senior and family life, because the difficult to realize he diary activities create great impact in the familiar structure. The domiciliary assistance cooperates for humanization of the care, effectiveness and more participation. The care support and health education are primordial to orient and help it in the more difficult situations.

Key words: Care. Dependent sênior. Family. Aging. Senior's health.

\section{Referências}

BRUM, A. K. R.; TOCANTINS, F. R.; SILVA, T. J. E. S. S. O Enfermeiro como instrumento de ação no cuidar do idoso. Revista LatinoAmericana de Enfermagem, São Paulo, v. 13, n. 6, p. 1019-1026, 2005.

CALDAS, C. P. Envelhecimento com dependência: responsabilidades e demandas da família. Cadernos de Saúde Pública, Rio de Janeiro, v. 19, n. 3, p. 773-781, 2003.

CREUTZBERG, M. tratar mais a pessoa idosa, sobre tudo a que está acamada: subsídios para o cuidado domiciliar. Mundo da Saúde, Brasília, v. 24, n. 4, p. 298-305, 2000.

ELSEN, I.; MARCON, S. S.; SANTOS, M. R. $O$ viver em família e sua interface com a saúde e a doença. Maringá: Eduem, 2002.

GONÇALVES, L. H. T. et. al. Perfil da família cuidadora de idoso doente/fragilizado do contexto sociocultural de Florianópolis, SC. Texto \& Contexto em Enfermagem, Florianópolis, v. 15, n. 4, p. 570-577, 2006.

INSTITUTO BRASILEIRO DE GEOGRAFIA E ESTATÍSTICA. Pesquisa Nacional por Amostra de Domicílios. Dados sobre População do Brasil. Rio de Janeiro: IBGE, jul. 2002.

KARSCH, U. M. Idosos dependentes: famílias e cuidadores. Cadernos de Saúde Pública, Rio de Janeiro, v. 19, n. 3, p. 861-866, 2003.

LEAL, M. G. S. O Desafio da longevidade e o suporte ao cuidador. Revista da Terceira Idade, São Paulo, v. 11, n. 20, p. 19-29, 2000.

LEME, L. E. G.; SILVA, P. S. C. P. O idoso e a família. In: PAPALEU NETTO, M. (Org.). Gerontologia: a velhice e o envelhecimento 
em visão globalizada. São Paulo: Atheneu, 2002. p. 92-97.

RODRIGUES, S. L. A.; WATANABE, H. A. W.; DERNTL, A. M. A saúde de idosos que cuidam de idosos. Revista Escola de Enfermagem da Universidade de São Paulo, São Paulo, v. 40, n. 4, p. 493-500, 2006.

SMELTZER, S. C; BARE, B. G. Tratado de enfermagem médico-cirúrgico. 10. ed. Rio de Janeiro: Guanabara Koogan, 2005.

SOUZA, L. F. F.; VAZ, M. S. C. Cuidar da $3^{\mathbf{a}}$ idade: um desafio no cotidiano hospitalar. Enfermagem Atual, Rio de Janeiro, v. 40, n. 1, p. 30-36, 2007.

SUDDARTH, D. S. et al. Brunner \& Suddarth: tratado de enfermagem médico-cirúrgica. Rio de Janeiro: Guanabara Koogan, 2005.

THOBER, E.; CREUTZBERG, M.; VIEGAS, K. Nível de dependência de idosos e cuidados no âmbito domiciliar. Revista Brasileira de Enfermagem, Brasília, v. 58, n. 4, p. 438-443, 2005. 